\title{
Enhanced Survival and Regeneration of Axotomized Retinal Ganglion Cells by a Mixture of Herbal Extracts
}

\author{
ZELDA H. CHEUNG, ${ }^{1,2}$ KWOK-FAI SO,${ }^{1,2,5}$ QIANG LU, ${ }^{1}$ HENRY K. YIP, ${ }^{1,2}$ WUTIAN WU,, 2 \\ JACQUELINE J. SHAN, ${ }^{2,3}$ PETER K.T. PANG, ${ }^{2}$ and CHIEH-FU CHEN, ${ }^{2,4}$
}

\begin{abstract}
The aim of this study is to investigate the effects of Panax quinquefolius L. extract (PQE), Ginkgo biloba extract (GBE), and Hypericum perforatum extract (HPE), in combination or alone, on the survival and regeneration of axotomized retinal ganglion cells (RGCs) in an optic nerve transection model in adult hamsters. Unilateral transection of the optic nerve was performed to evaluate the effects of herbal extracts on the survival of axotomized RGCs. Effects of the herbal extracts on axonal regeneration of axotomized RGCs, on the other hand, were studied by attaching a peripheral nerve graft onto the transected ocular stump to induce regeneration. Operated animals received daily oral administration of vehicle or herbal extracts (PQE, GBE, and HPE), alone or in combination, for 7 and 21 days, respectively, in the survival and regeneration experiments. Surviving and regenerating RGCs were retrogradely labeled with Fluoro-Gold. The eyes were then enucleated and the retinas were flat-mounted for the counting of the labeled RGCs. Treatment with PQE, GBE and HPE alone failed to offer neuroprotection to injured RGCs. However, treatment with Menta-FX, a mixture of PQE, GBE, and HPE, significantly augmented RGC survival 7 days postaxotomy. Treatment with Menta-FX also induced a significant $(87 \%)$ increase in the number of regenerating RGCs 21 days after optic nerve transection. This study demonstrates that herbs can act as a potential neuroprotective agent for damaged RGCs. It also suggests that the therapeutic value of herbal remedies can be maximized by the use of mixtures of appropriate herbs.
\end{abstract}

Key words: Ginkgo biloba; Hypericum perforatum; neuroprotection; optic nerve; Panax quinquefolius L.

\section{INTRODUCTION}

$\mathbf{T}$ RANSECTION OF THE OPTIC NERVE in adult rats induces neuronal death in approximately $80 \%$ of the retinal ganglion cells (RGCs) in 14 days (Villegas-Perez et al.,
1993; Peinado-Ramon et al., 1996; Berkelaar et al., 1994). It was proposed that the retrograde degeneration of axotomized RGCs is due to a lack of trophic factor supply upon disconnection from their target sites. This view is supported by experiments showing trophic fac-

\footnotetext{
${ }^{1}$ Department of Anatomy, Faculty of Medicine, University of Hong Kong, Hong Kong, China.

${ }^{2}$ International Research and Development Program on Traditional Chinese and Natural Medicine, University of Hong Kong, Hong Kong, China.

${ }^{3} \mathrm{CV}$ Technologies, Edmonton, Alberta, Canada.

${ }^{4}$ National Research Institute of Chinese Medicine, Taiwan.

${ }^{5}$ Central Laboratory of the Institute of Molecular Technology for Drug Discovery and Synthesis, University of Hong Kong.
} 
tors, including BDNF (Yan et al., 1999; Isenmann et al., 1998; Peinado-Ramon et al., 1996; Di Polo et al., 1998); GDNF (Yan et al., 1999); CNTF (Mey and Thanos, 1993; Yip et al., 2000); NGF (Lambiase et al., 1997; Rabacchi et al., 1994b); and NT4/5 (Peinado-Ramon et al., 1996; Yip and So, 2000) are able to delay RGC death following axotomy. Recent evidence has attributed the death of axotomized RGCs to apoptosis (Kermer et al., 1999b; Rabacchi et al., 1994a; Garcia-Valenzuela et al., 1994). Interruption of the apoptotic pathway with agents like caspase inhibitor z-DEVD-cmk (Kermer et al., 1998, 1999a) or overexpression of bcl-2 in transgenic mice (Cenni et al., 1996; Porciatti et al., 1996; Bonfanti et al., 1996) augment the survival of axotomized RGCs. Oxidative stress has been implicated as one of the potent inducers and modulators of the apoptotic pathway in ischemia (Keller et al., 1998) and NMDA-induced retinal toxicity (Vorwerk et al., 1997; Castagne et al., 1999). Thus, it is not unreasonable to speculate that antioxidants may offer neuroprotection to axotomized RGCs. In support of this hypothesis, intravitreal injections of the nitric oxide synthase (NOS) inhibitors, L-NAME and L-NOLA, enhance the survival of RGCs following axotomy (Ball and Keoberle, 1999). These observations suggest that free radicals play a role in the etiology of axotomized RGC death, and thus, free radical scavengers may be neuroprotective for axotomized RGCs.

Extracts from American ginseng (Panax quinquefolius L.; Kitts et al., 2000), Ginkgo biloba (Baudouin et al., 1999; Wei et al., 2000; Bastianetto et al., 2000a) and St. John's Wort (Hypericum perforatum; Tripathi and Pandey, 1999) have been demonstrated to exhibit antioxidant properties. In addition, these herbal extracts appear to offer neuroprotection in other models of injury. For example, saponins of Panax quinquefolius L. have been shown to delay neuronal death during ischemia (Attele et al., 1999; Wen et al., 1996) and glutamate-induced excitotoxicity (Kim et al., 1998). Ginkgo biloba has also been observed to protect against ischemic injury (Pierre et al., 1999), $\beta$-amyloid-induced toxicity (Bastianetto et al., 2000a), nitric oxide-induced toxicity (Bastianetto et al., 2000b), MPTP-induced nigrostriatal neuronal death (Wu and Zhu, 1999), and light-induced photoreceptor damage (Ranchon et al., 1999). Taken together, these studies suggest that Ginkgo biloba, Panax quinquefolius $L$., and Hypericum perforatum extracts may have neuroprotective effects in axotomy-induced RGC death. In the current communication, we examine the effects of these extracts, alone or in combination, on the survival of axotomized RGCs in adult hamsters. Since robust regeneration of damaged axons also requires survival of the parental neurons, agents that increase viability of axotomized RGCs may also promote the regrowth of their axons. Accordingly, we also investigate the effects of these herbal extracts on the capacity of axotomized RGCs to regenerate into a peripheral nerve graft, which provides a permissive environment for axonal regeneration (So and Aguayo, 1985).

\section{MATERIALS AND METHODS}

\section{Animals}

A total of 112 adult golden hamsters (Mesocricetus auratus, 6-8 weeks old, weighing 60-80 g) were used in this study. Golden hamsters have been used in studies in which peripheral nerve grafts are attached to the ocular stump to induce long-distance regeneration (So and Yip, 2001; Cui et al., 1999). The ability of the regenerated axons to form functional synapses has also been demonstrated by connecting the peripheral nerve grafts to targets such as the superior colliculus (Sauve et al., 2001; Sasaki et al., 1999). Specific tests utilizing the orienting behavior exhibited by hamsters can then be performed to accurately examine functional recovery following axotomy (Carman and Schneider, 1992; Schneider, 1969). The use of golden hamsters in this study therefore allows future investigation of the herbal extracts' ability to augment and restore visual performances. All operations were carried out in animals anesthetized with intraperitoneal injection of sodium pentobarbitone (Nembutal, Rhone Merieux Australia Pty Ltd., Australia; 50 mg/kg). Animals were weighed on the day of operation and on the day of sacrifice. The incidence of diarrhea and acute weight loss were monitored as indicators of the herbal extracts potential side effects as adverse reactions such as gastrointestinal problems, diarrhea and acute weight loss have been associated with HPE or PQE intake (Ernst et al., 1998; Vogler et al., 1999). All animals were sacrificed with an overdose of anesthesia.

\section{Extracts}

Herbal extracts from CV Technologies (Canada, Edmonton, Alberta) were used in this study. The extracts were standardized by their biological activities and chemical compositions using ChemBioPrint ${ }^{\mathrm{TM}}$ technology, ensuring batch-to-batch consistency. Three extracts were used: (1) extract from the root of Panax quinquefolius $L$. (PQE), standardized to contain $25 \%$ ginsenosides; (2) Ginkgo biloba extract (GBE), standardized to contain $24 \%$ flavonoid glycosides and 6\% terpenoids; and (3) $\mathrm{Hy}$ pericum perforatum extract (HPE), standardized to contain $0.3 \%$ hypericin. Two herbal mixtures were constructed from these standardized extracts: (1) AD-FX, consisted of $80 \%$ PQE and $20 \%$ GBE by weight; and (2) 
Menta-FX, composed of $30.8 \%$ PQE, $61.5 \%$ HPE and $7.7 \%$ GBE by weight. All herbal potions (single extracts or mixtures) were suspended in sterile $0.01 \mathrm{M}$ phosphatebuffered saline (PBS; pH 7.4) at concentrations specified in the following section, stored at $4^{\circ} \mathrm{C}$ and administered to animals within 2 weeks. The extracts were delivered directly into the esophagus of the animals with a specialized feeding cannula.

\section{Herbal Extracts Treatment Scheme}

In the survival studies, animals were divided into three groups: control (0.01 M PBS, $n=10)$, single extracts treatment $(n=53)$, and extract-mixtures treatment $(n=$ $21)$. Refer to Table 1 for detailed description of the treatment groups. Briefly, the single extract treatment group was divided into three subgroups. Animals received either PQE $(10,20$, or $30 \mathrm{mg})$, GBE $(2,6$, or $12 \mathrm{mg})$, or HPE $(10,20$, and $30 \mathrm{mg})$. The extract-mixture treatment group was divided into two subgroups: $30 \mathrm{mg}$ AD-FX and $30 \mathrm{mg}$ Menta-FX. Animals were fed daily with 0.5 $\mathrm{mL}$ of vehicle or herbal extracts starting on the day of operation for 7 days. Animals were allowed to survive for 7 days postaxotomy before they were sacrificed.
In the regeneration studies $(n=23)$, animals were divided into four groups (Table 1). Animals were fed with $0.5 \mathrm{~mL}$ of vehicle (0.01 M PBS), $30 \mathrm{mg}$ PQE, $30 \mathrm{mg}$ AD-FX, or $30 \mathrm{mg}$ Menta-FX per day for 21 days, starting on the day of operation. Animals were allowed to survive for 21 days postgrafting before they were sacrificed.

\section{Optic Nerve Transection and Attachment of Peripheral Nerve Graft}

In the survival studies $(n=84)$, unilateral optic nerve transection was performed. A skin incision was made close to the superior orbital rim of the left eye. The left orbita was exposed and the superior extraocular muscles were excised to uncover the optic nerve. After opening the dural sheath, the optic nerve was completely transected at about $1.5 \mathrm{~mm}$ from the optic disc.

In the regeneration studies, an autologous peripheral nerve (PN) graft was attached to the ocular stump as a conduit for axonal regeneration. The optic nerve was transected $0.5 \mathrm{~mm}$ from the optic disc. A segment of the sciatic nerve $(1 \mathrm{~cm})$ was sutured onto the proximal stump of the optic nerve with a $10 / 0$ suture. The distal end of the PN graft was secured on the connective tissue of the

Table 1. Treatment Paradigm Employed to Investigate the Effects of PQE, HPE, And GBE Alone or in Combination on the Survival and Regeneration of Axotomized RGCs

\begin{tabular}{|c|c|c|c|c|c|}
\hline Experiment & Groups & $P Q E(m g)$ & $G B E(m g)$ & $H P E(m g)$ & No. of animals \\
\hline \multicolumn{6}{|l|}{ Survival } \\
\hline & Control (PBS) & & & & 10 \\
\hline & PQE single extract & & & & \\
\hline & & 10 & & & 6 \\
\hline & & 20 & & & 6 \\
\hline & & 30 & & & 9 \\
\hline & GBE single extract & & & & \\
\hline & & & 2 & & 5 \\
\hline & & & 6 & & 5 \\
\hline & & & 12 & & 5 \\
\hline & HPE single extract & & & & \\
\hline & & & & 10 & 6 \\
\hline & & & & 20 & 6 \\
\hline & & & & 30 & 5 \\
\hline & Extract-mixtures & & & & \\
\hline & $30 \mathrm{mg}$ AD-FX & 24 & 6 & & 10 \\
\hline & $30 \mathrm{mg}$ Menta-FX & 9.23 & 2.31 & 18.46 & 11 \\
\hline \multicolumn{6}{|l|}{ Regeneration } \\
\hline & Control (PBS) & & & & 6 \\
\hline & PQE single extract & 30 & & & 6 \\
\hline & $30 \mathrm{mg}$ AD-FX & 24 & 6 & & 6 \\
\hline & $30 \mathrm{mg}$ Menta-FX & 9.23 & 2.31 & 18.46 & 5 \\
\hline
\end{tabular}

The respective amount of each of the single extracts in $30 \mathrm{mg}$ of AD-FX and Menta-FX is listed. 
skull using a 5/0 suture (So and Aguayo, 1985). Care was taken to keep the blood supply to the retina intact throughout the operations. Animals with compromised blood supply after the operation were discarded.

\section{Retrograde Labeling of Retinal Ganglion Cells and Labeled Retinal Ganglion Cells Counts}

To assess the number of RGCs in normal retina, the optic nerve was transected in five animals. A Gelfoam (Upjohn, Kalamazoo, MI) soaked with 6\% Fluoro-Gold (FG; Fluorochrome, Denver, CO) was placed at the cut site immediately after transection to label the RGCs. The animals were sacrificed 2 days later. Since optic nerve transection results in delayed cell death beginning on day 4 after axotomy (Berkelaar et al., 1994), the number of RGCs labeled was assumed to be representative of the number of RGCs present in normal retinas.

In the survival studies, surviving RGCs were retrogradely labeled by placing a Gelfoam soaked with $6 \%$ FG at the ocular stump 2 days before sacrificing the animals. In the regeneration experiment, regenerating RGCs were labeled by injecting $1.5 \mu \mathrm{L}$ of $6 \%$ FG into the distal end of the PN graft 3 days before the animals were killed. The left eye of the euthanatized animals in the normal, survival, and regeneration groups were enucleated, and the retinas dissected in 4\% paraformaldehyde (PFA). Four radial cuts were made to divide the retinas into four quadrants (superiotemporal, superionasal, inferiotemporal and inferionasal). The retinas were then postfixed in 4\% PFA for 45 min before they were flat-mounted with the RGC side up on gelatin-coated slides in $30 \%$ glycerin.

The slides were examined under fluorescence microscopy using an ultra-violet filter (excitation wavelength $=330-380 \mathrm{~nm}$ ). Labeled RGCs were counted along the median line of the four quadrants starting from the optic disc to the peripheral border of the retina at 500 $\mu \mathrm{m}$ intervals, under an eyepiece grid of $200 \times 200 \mu \mathrm{m}$. The mean density of labeled RGCs in each retina was then multiplied by the area of the retina to obtain the number of labeled RGCs in the retina.

All data were expressed as mean \pm SD. Statistical significance was evaluated by one way ANOVA, followed by Tukey-Kramer post hoc test. Differences were considered significant for $p<0.05$. Retinas that were poorly labeled were excluded from statistical analysis.

\section{RESULTS}

\section{Effects of Optic Nerve Transection on the} Number of Retinal Ganglion Cells

The number of RGCs labeled in normal retina was $65,890 \pm 5,684$. Optic nerve transection led to a marked decrease in the number of surviving RGCs 7 days after axotomy in the PBS-treated group $(21,841 \pm 3,536$; Fig. $1)$. The morphology of the labeled RGCs was in accordance with that observed in previous studies (PeinadoRamon et al., 1996; Koeberle and Ball, 1998). FG fluorescence was found in the somata and proximal dendrites of the RGCs (Fig. 2A). In addition to the labeled RGCs, microglia, characterized by their small spindle-shaped soma could also be identified (arrowhead, Fig. 2A).

Effects of Single Extracts of $P Q E, G B E$, and HPE on the Survival of Axotomized Retinal Ganglion Cells

Oral administration of PQE, GBE, and HPE for 7 days did not adversely affect the animals well-being. No diarrhea or acute weight loss was observed (data not shown). For the three dosages of PQE examined, treatment with $10 \mathrm{mg}(23,999 \pm 3,099), 20 \mathrm{mg}(23,634 \pm 6,659)$, or 30 mg $(23,984 \pm 6,362)$ daily for 7 days had negligible effects on axotomized RGC survival (Fig. 1). Daily administration of $10 \mathrm{mg}(21,735 \pm 3,074), 20 \mathrm{mg}$ $(23,928 \pm 4,841)$, and $30 \mathrm{mg}$ of HPE $(25,044 \pm 2,634)$ resulted in slight enhancement of the number of surviving RGCs that appeared to be dose-related. The increase, however, was not significant (Fig. 1). Treatment with 2 $\mathrm{mg}(24,434 \pm 1,692), 6 \mathrm{mg}(23,065 \pm 3,883)$, and $12 \mathrm{mg}$ of $\operatorname{GBE}(24,861 \pm 4,167)$ similarly did not significantly augment the number of surviving RGCs at 7 days after axotomy (Fig. 1).

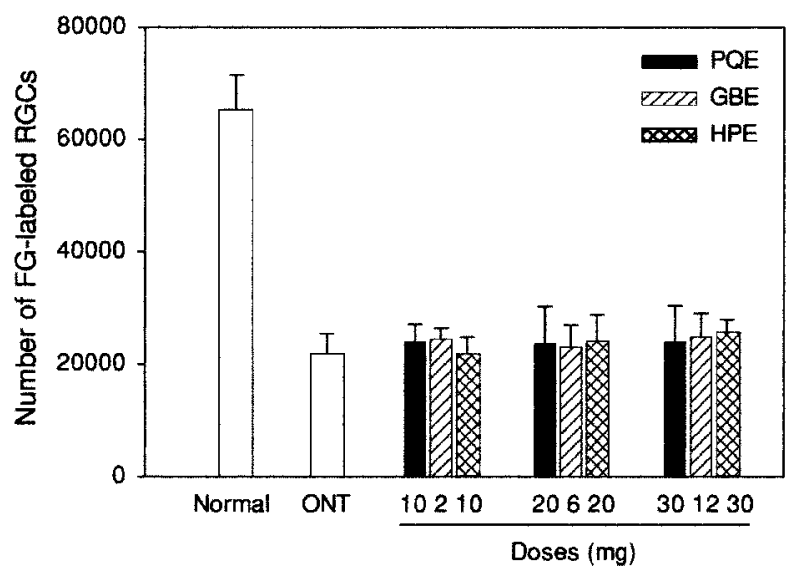

FIG. 1. Effects of Panax quinquefolius $L$. extract (PQE; 10, 20, and $30 \mathrm{mg}$ ), Ginkgo biloba extract (GBE; 2, 6, and $12 \mathrm{mg}$ ), and Hypericum perforatum extract (HPE; 10, 20, and $30 \mathrm{mg}$ ) on the survival of axotomized retinal ganglion cells 7 days after axotomy. Note that none of the treatment significantly affected the number of surviving cells compared with that of the PBS-treated optic nerve transection (ONT) group. 

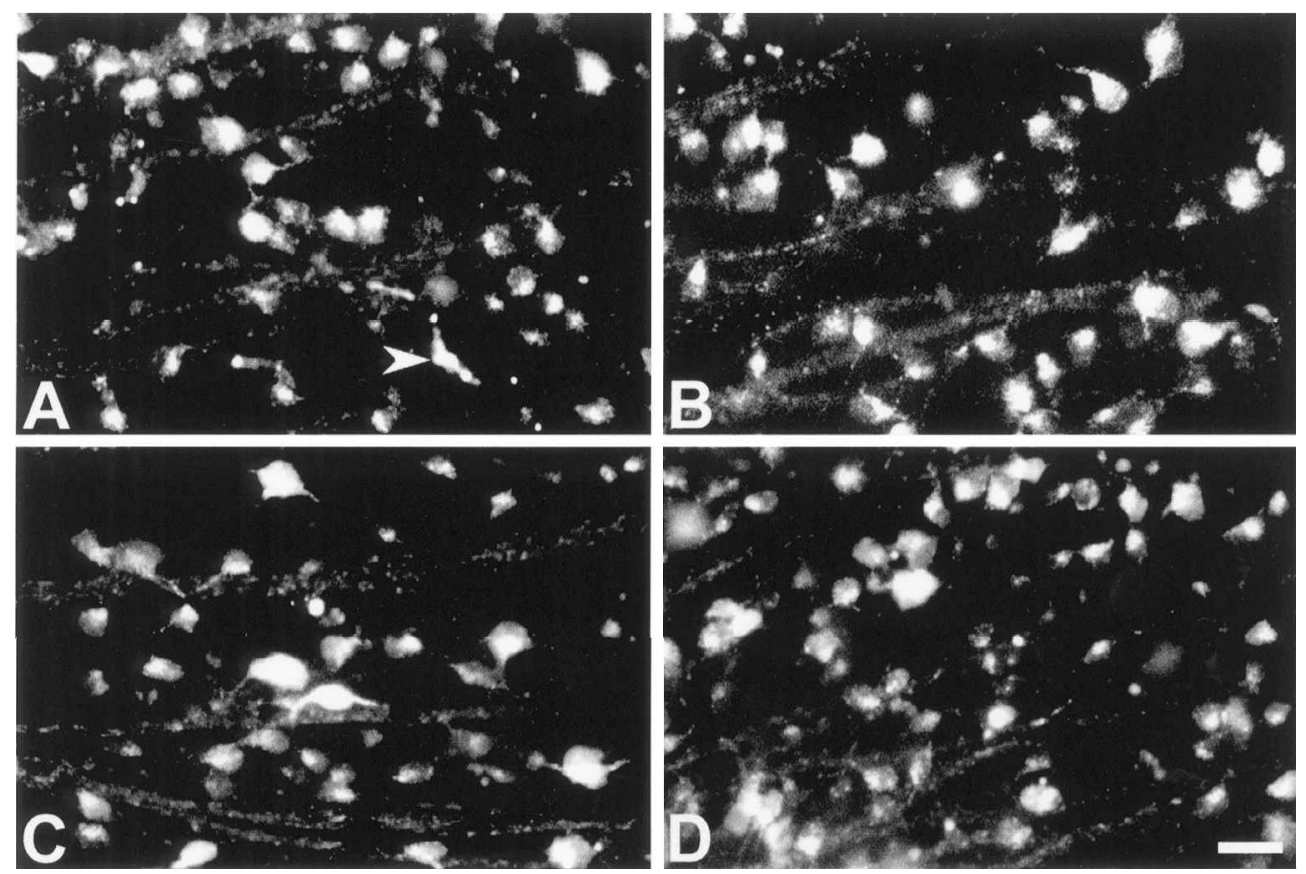

FIG. 2. Photomicrographs of FG-labeled RGCs taken from retina treated with vehicle (A) or herbal extracts (B-D) at comparable distance from the optic disc. (A) Seven days after optic nerve transection, very few labeled RGCs remained. Notice the presence of microglia (arrowhead), which is characterized by a spindle-shaped somata. (B) Treatment with $30 \mathrm{mg}$ of PQE daily for 7 days has negligible effect on the number of labeled RGCs. (C) Treatment with AD-FX (30 mg/day) again has limited neuroprotective effect on the axotomized RGCs. (D) Treatment with Menta-FX (30 mg/day) lead to a marked increase in the number of surviving RGCs 7 days post axotomy. Bar $=25 \mu \mathrm{m}$.

\section{Effects of $A D-F X$ and Menta-FX on the Survival of Axotomized Retinal Ganglion Cells}

No adverse reaction was observed following oral administration of AD-FX and Menta-FX for 7 days, with no incidence of diarrhea or acute weight loss (data not shown). Treatment with $30 \mathrm{mg}$ of AD-FX per day (Fig. $2 \mathrm{C})$ caused a slight increase in the number of surviving RGCs (27,034 $\pm 4,268$; Fig. 3), although the enhancement was not statistically significant. However, daily treatment with $30 \mathrm{mg}$ of Menta-FX (Fig. 2D) significantly augmented the number of surviving RGCs $(30,385 \pm$ 6,187 ; Fig. 3), rescuing $19 \%$ of the RGCs that would have died without treatment ( $p<0.01$; one-way ANOVA). Rescue rate was estimated by the following formula: [(no. of RGCs in treatment group - no. of RGCs in ONT group)/(no. of RGCs in normal retina - no. of RGCs in ONT group)] $\times 100$. Administration of PQE, HPE, and GBE individually had negligible effects on axotomized RGC survival, in contrast to the significant neuroprotection provided by a mixture of the three at equivalent dosages.

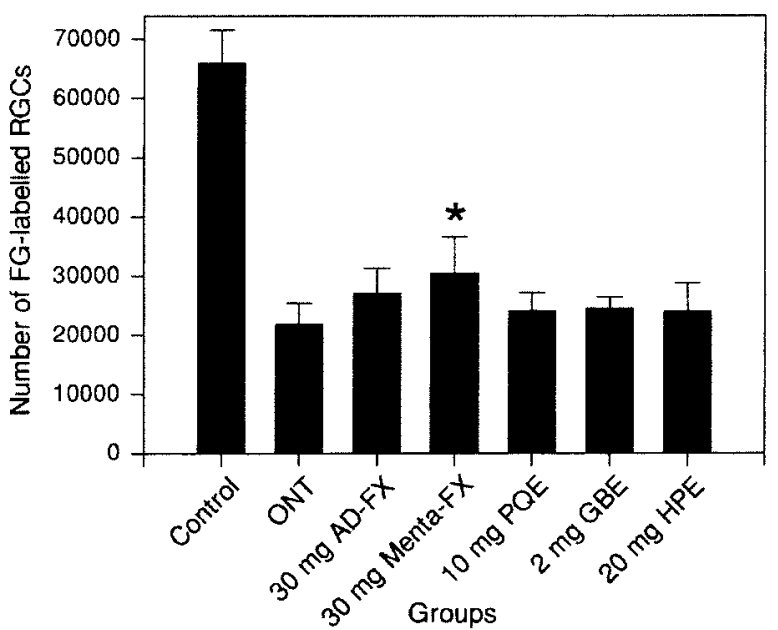

FIG. 3. Effects of herbal extracts mixtures on the survival of axotomized retinal ganglion cells 7 days after axotomy. Note that while equivalent dosages of PQE, GBE, and HPE failed to increase the number of surviving cells, Menta-FX (a mixture of the three), offered significantneuroprotection. ${ }^{*} p<0.01$ versus optic nerve transection (ONT). 
Effects of PQE, AD-FX, and Menta-FX on Regeneration of Axotomized Retinal Ganglion Cells into Peripheral Nerve Graft

Attachment of PN graft to the optic nerve stump elicited approximately $5 \%$ of the RGCs to regrow their axons into the PN graft 21 days after axotomy $(3,301 \pm$ 1,192; see Fig. 5 below). As seen in Figure 4A, regenerating RGCs exhibited a characteristic hypertrophic morphology (Cui et al., 1999) that was drastically different from the surviving RGCs observed in Figure 2A. Treatment with $30 \mathrm{mg} /$ day PQE $(3,702 \pm 637)$ and $30 \mathrm{mg} /$ day AD-FX $(4,658 \pm 1,348)$ had no significant effects on the regenerative capability of the axotomized RGCs. Treatment with Menta-FX (Fig. 4D), however, led to a significant increase in number of regenerating RGC $(6,174 \pm 1,966$; Fig. 5$)$. This is a $87 \%$ enhancement in the number of regenerated RGCs compared with control $(p<0.05$, one-way ANOVA).

\section{DISCUSSION}

In this study, we examined the effects of herbal extracts on axotomized RGCs. Our data showed that negligible neuroprotection was offered by $\mathrm{PQE}, \mathrm{GBE}$, and
HPE treatment, despite previously demonstrated free radical scavenging capacity of the extracts in earlier studies. The apparent lack of action by these extracts could be due to poor absorption of extract components, although earlier studies suggest that HPE and GBE are well absorbed after ingestion (Franklin et al., 1999; Ranchon et al., 1999). In addition, previous studies using oral administration of Panax quinquefolius L. saponins and EGb761 (a Ginkgo biloba extract) have shown that there is significant neuroprotection of damaged neurons in vivo (Wen et al., 1996; Pierre et al., 1999; Baudouin et al., 1999; Ranchon et al., 1999), implying that the extracts components were absorbed and remained active.

Insufficient amounts of extract metabolites reaching the eye and/or the injury site may also result in the lack of neuroprotection by single extracts. The concentration of extract components required at the site of injury to rescue damaged neurons can vary depending on the type of injury and neuronal subtypes. For example, previous studies using lower doses of GBE than that adopted in the current study have been shown to provide effective neuroprotection in light-damaged photoreceptors (Ranchon et al., 1999) and ischemic cortical neurons (Pierre et al., 1999). Despite the higher doses of GBE used in the present study, no protective effect was observed.
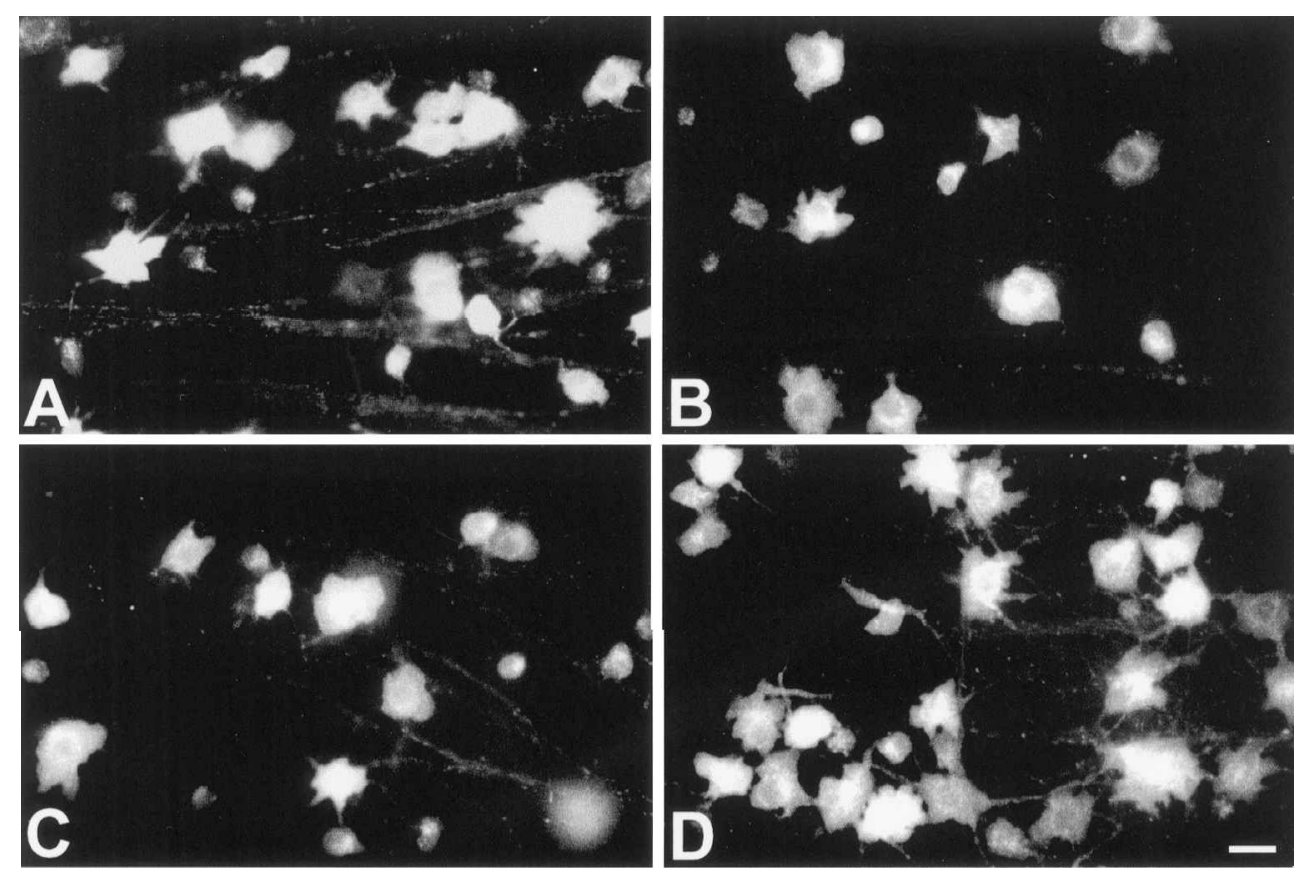

FIG. 4. Photomicrographs of FG-labeled RGCs taken from retina treated with vehicle (A) or herbal extracts (B-D) at comparable distance from the optic disc. (A) Twenty-one days after peripheral nerve graft attachment, about $5 \%$ of the RGCs extended their axons. (B) Treatment with $30 \mathrm{mg}$ of PQE daily for 21 days has negligible effect on the number of regenerated RGCs. (C) Treatment with AD-FX (30 mg/day) again has no effect on the regenerative capacity of the axotomized RGCs. (D) Treatment with Menta-FX (30 mg/day) lead to a marked increase in the number of regenerating RGCs 21 days postgrafting. Bar $=25 \mu \mathrm{m}$. 


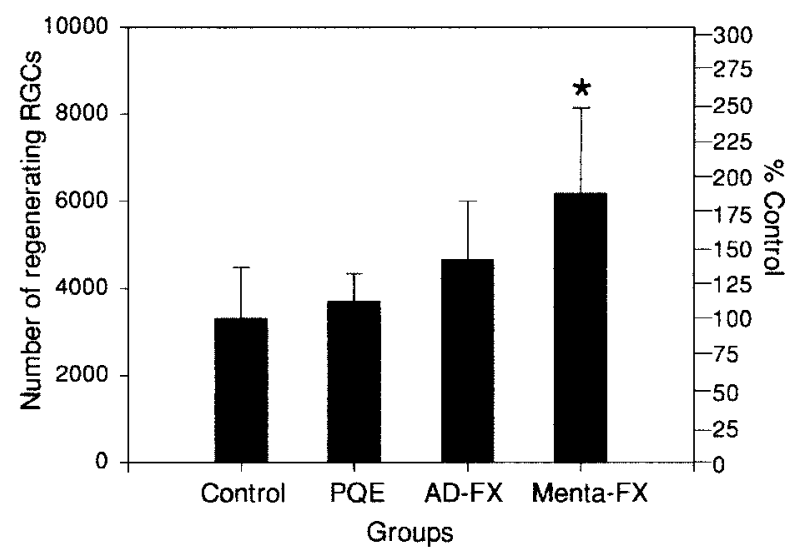

FIG. 5. Effects of Panax quinquefoliusL. extract (PQE), ADFX, and Menta-FX on the number of regenerating RGCs into attached peripheral nerve graft. ${ }^{*} p<0.05$ versus control.

Therefore, it is possible that a higher concentration of antioxidants is needed at the site of injury to protect RGCs from axotomy-induced death.

Other factors contributing to the lack of neuroprotection by single extracts may include the low concentration or absence of antioxidative ingredients in the extract preparations used in this study. The amount of active components in a herbal extract can vary depending on the strain of the herbs used. Different strains of ginseng, for example, have been demonstrated to contain different amounts of various ginsenosides (Chan et al., 2000; Wang et al., 1999). The PQE preparation used in this study may hence lack or contain lower concentrations of various ginsenosides isolated from Panax ginseng, which have been demonstrated to be neuroprotective against ischemia (Attele et al., 1999; Wen et al., 1996) and glutamate-induced excitotoxicity (Kim et al., 1998) in neurons.

Finally, the treatment paradigm employed can also affect the effectiveness of the herbal extracts. Some of the previous studies with Ginkgo biloba extract involved pretreatment or co-treatment of the damaged neurons. However, in this study, treatment with PQE, HPE, and GBE did not commence until at least $2 \mathrm{~h}$ after the damage had been inflicted. This difference may contribute to the observed lack of neuroprotective effect of single extracts on axotomized RGCs. It should be noted, however, that studies by Bastianetto et al. have shown that post-injury treatment with EGb 761 can rescue neurons from nitric oxide-induced cell death (Bastianetto et al., 2000b) and $\beta$-amyloid-induced toxicity (Bastianetto et al., 2000a). The time frame in which treatment need to commence may vary with the type of damage and neuronal subtypes. Experiments using a pretreatment paradigm will be helpful in resolving this problem.
A significant finding of this study is the neuroprotective effect exhibited by Menta-FX. The observation that the herbal extracts were only effective in combination suggests that the therapeutic values of herbal extracts can be maximized by mixing appropriate herbs together. Indeed, potency of a herbal remedy is often increased by using the whole extract, including the active ingredients and the supposedly inactive ingredients. For example, a study by Wu and Zhu (1999) has shown that EGb 761 prevents the decrease in dopamine levels in striatum following MPTP injection while the isolated compounds of EGb 761, ginkgolides A and B, do not. In addition, only ginseng extract can protect against ischemic neuronal death in gerbils, with ginsenosides $\mathrm{Rb}_{1}, \mathrm{Rg}_{1}$, and $\mathrm{R} 0$ being less effective or having no effects at all on mitigating the damage (Wen et al., 1996).

The mechanisms involved in the neuroprotective effects of Menta-FX remain unknown. The neuroprotective property of Menta-FX may be a manifestation of the summation of free radical scavenging abilities of the different extracts. This is unlikely, however, given that high doses of PQE, GBE and HPE failed to provide equivalent neuroprotection offered by Menta-FX. On the other hand, Menta-FX may provide other neuroprotective properties aside from anti-oxidative capacity. Other potential mechanisms include (1) generation of novel neuroprotective compounds via chemical reactions among the ingredients of the extracts and (2) active compounds in the different extracts may act on different components of the apoptotic pathway involved in the death of axotomized RGCs. Menta-FX may directly inhibit activation of caspase-3 or -9 , which have been demonstrated in axotomized RGCs (Kermer et al., 1999b). Menta-FX may also offer neuroprotection by up-regulating production of trophic factors shown to enhance survival of axotomized RGCs, such as BDNF or CNTF (Yan et al., 1999; Isenmann et al., 1998; Peinado-Ramon et al., 1996; Di Polo et al., 1998; Mey and Thanos, 1993). It is difficult to identify the pathways involved in the rescue effect of Menta-FX since the pharmacology of these extracts on injured neurons has not been completely elucidated. However, our observations suggest that two factors may play a role in Menta-FX's neuroprotective property. Note that AD-FX, a mixture of PQE and GBE, differs from Menta-FX, a mixture of PQE, HPE, and GBE, by the exclusion of HPE. The lack of significant rescue effect by AD-FX may suggest that (1) HPE is essential for the neuroprotective property of Menta-FX; or (2) all three of the extracts are required for the neuroprotective capability observed in Menta-FX. It remains to be clarified whether other combinations of the three herbal extracts such as HPE and GBE; or PQE and HPE, contribute to the neuroprotective effects of Menta-FX. 
In this study, treatment with PQE and AD-FX had negligible effects on the regeneration of axotomized RGCs. Treatment with Menta-FX, on the other hand, almost doubled the number of regenerating RGCs compared to that from the PBS-treated group $(9.4 \%$ versus $5 \%$ of total RGCs). The mechanisms underlying Menta-FX's ability to augment regeneration of RGCs into a peripheral nerve graft are possibly related to its capacity to rescue axotomized RGCs. CNTF (Mey and Thanos, 1993; Yip et al., 2000; Cui et al., 1999) and aurintricarboxylic acid (Heiduschka and Thanos, 2000) have been demonstrated recently to enhance both survival and regeneration of axotomized RGCs. Similarly, Menta-FX may promote regeneration by increasing the number of surviving cells, hence permitting the regeneration-sensitive subpopulations of RGCs to survive long enough to extend their axons. PQE and AD-FX, which failed to significantly mitigate axotomy-induced RGC death also lacked the ability to enhance regeneration of axotomized RGCs. On the other hand, Menta-FX may also augment regeneration by modulating other parameters known to affect regenerative capacity. For example, Menta-FX may upregulate the expressions of proteins such as GAP-43, which was shown to precede robust regeneration of transected axons (Ng et al., 1995). Menta-FX may also promote the synthesis of cytoskeletal components in axotomized RGCs to allow effective regeneration. In addition, it may change the behavior of growth cones to favor long-distance regeneration over limited local sprouting. Extrinsically, Menta-FX may neutralize the inhibitory environment surrounding the growing axons, such as the glial scar, to enable efficient regrowth of the axons (Grill and Tuszynski, 1999). However, the precise actions of MentaFX on these pathways remain to be uncovered by further experimentation.

In conclusion, the potential use of herbal extracts as neuroprotective agents for the injured RGCs is encouraging. Herbal extracts can be taken orally and possess minimal side effects. This is a significant advantage compared to drugs that are effective only when administered via an invasive route. In addition, the effectiveness of Menta-FX as a neuroprotective agent may be further increased by modifying the dosage used or the proportion of PQE, GBE and HPE in the mixture. Recent findings from our lab show that higher doses of Menta-FX further enhance the number of surviving RGCs at various time-points following axotomy. In addition, the duration of significant neuroprotection offered by Menta-FX can be extended using higher doses (unpublished observations). This suggests possible long-term neuroprotection by the herbal mixture.

This study holds important implications on the approach to take to enhance the therapeutic values of herbal extracts in neurodegenerative diseases or central nervous system trauma. We have demonstrated that a herbal extract-mixture exhibits neuroprotective effects on axotomized RGCs and increases regenerative propensity of the damaged axons. In addition, we showed that the therapeutic capacity of the herbal extracts is significantly augmented by using mixtures of the herbal extracts. We have established an easily-accessible and reproducible model for further investigations of the neuroprotective effect of these herbs. Fully explicating the precise interaction of these herbs will increase our understanding of the herbs and the mechanisms involved in the death \& survival of axotomized RGCs.

\section{ACKNOWLEDGMENTS}

We would like to thank Dr. Margaret Tan for her critical review of the manuscript. This research is supported by the International Research and Development Program on Traditional Chinese and Natural Medicine, the University of Hong Kong, and a research grant from the National Research Institute of Chinese Medicine, Taiwan. Z.H.C. is supported by a Croucher Studentship, Hong Kong.

\section{REFERENCES}

ATTELE, A.S., WU, J.A., and YUAN, C.S. (1999). Ginseng pharmacology: multiple constituents and multiple actions. Biochem. Pharmacol. 58, 1685-1693.

BALL, A.K., and KEOBERLE, P.D. (1999). Nitric oxide synthase inhibition delays axonal degeneration and promotes the survival of axotomized retinal ganglion cells. Exp. Neurol. 158, 366-381.

BASTIANETTO, S., RAMASSAMY, C., DORE, S., et al. (2000a). The Ginkgo biloba extract (EGb 761) protects hippocampal neurons against cell death induced by beta-amyloid. Eur. J. Neurosci. 12, 1882-1890.

BASTIANETTO, S., ZHENG, W.H., and QUIRION, R. (2000b). The Ginkgo biloba extract (EGb 761) protects and rescues hippocampal cells against nitric oxide-induced toxicity: involvement of its flavonoid constituents and protein kinase C. J. Neurochem. 74, 2268-2277.

BAUDOUIN, C., PISELLA, P.J., ETTAICHE, M., et al. (1999). Effects of EGb761 and superoxide dismutase in an experimental model of retinopathy generated by intravitreal production of superoxide anion radical. Graefes Arch. Clin. Exp. Ophthalmol. 237, 58-66.

BERKElAAR, M., ClARKE, D.B., WANG, Y.C., et al. (1994). Axotomy results in delayed death and apoptosis of retinal ganglion cells in adult rats. J. Neurosci. 14, 4368-4374.

BONFANTI, L., STRETTOI, E., CHIERZI, S., et al. (1996). 
Protection of retinal ganglion cells from natural and axotomyinduced cell death in neonatal transgenic mice overexpressing bcl-2. J. Neurosci. 16, 4186-4194.

CARMAN, L.S., and SCHNEIDER, G.E. (1992). Orienting behavior in hamsters with lesions of superior colliculus, pretectum, and visual cortex. Exp. Brain Res. 90, 79-91.

CASTAGnE, V., GAUTSCHI, M., LEFEVRE, K., et al. (1999). Relationships between neuronal death and the cellular redox status. Focus on the developing nervous system. Prog. Neurobiol. 59, 397-423.

CENNI, M.C., BONFANTI, L., MARTINOU, J.C., et al. (1996). Long-term survival of retinal ganglion cells following optic nerve section in adult bcl-2 transgenic mice. Eur. J. Neurosci. 8, 1735-1745.

CHAN, T.W., BUT, P.P., CHENG, S.W., et al. (2000). Differentiation and authentication of Panax ginseng, Panax quinquefolius, and ginseng products by using HPLC/MS. Anal. Chem. 72, 1281-1287.

CUI, Q., LU, Q., SO, K.F., et al. (1999). CNTF, not other trophic factors, promotes axonal regeneration of axotomized retinal ganglion cells in adult hamsters. Invest. Ophthalmol. Vis. Sci. 40, 760-766.

DI POLO, A., AIGNER, L.J., DUNN, R.J., et al. (1998). Prolonged delivery of brain-derived neurotrophic factor by adenovirus-infected Muller cells temporarily rescues injured retinal ganglion cells. Proc. Natl. Acad. Sci. U.S.A. 95, 3978-3983.

ERNST, E., RAND, J.I., BARNES, J., et al. (1998). Adverse effects profile of the herbal antidepressant St. John's wort (Hypericum perforatum L.). Eur. J. Clin. Pharmacol. 54, 589-594.

FRANKLIN, M., CHI, J., McGAVIN, C., et al. (1999). Neuroendocrine evidence for dopaminergic actions of hypericum extract (LI 160) in healthy volunteers. Biol. Psychiatry 46, 581-584.

GARCIA-VALENZUELA, E., GORCZYCA, W., DARZYNKIEWICZ, Z., et al. (1994). Apoptosis in adult retinal ganglion cells after axotomy. J. Neurobiol. 25, 431-438.

GRILL, R.J., and TUSZYNSKI, M.H. (1999). Axonal response to injury, in: CNS Regeneration: Basic Science and Clinical Advances (Tuszynski MH, Kordower JH, eds.) Academic Press: San Diego, pps. 27-53.

HEIDUSCHKA, P., and THANOS, S. (2000). Aurintricarboxylic acid promotes survival and regeneration of axotomised retinal ganglion cells in vivo. Neuropharmacology 39, 889-902.

ISENMANN, S., KLOCKER, N., GRAVEL, C., et al. (1998). Short communication: protection of axotomized retinal ganglion cells by adenovirally delivered BDNF in vivo. Eur. J. Neurosci. 10, 2751-2756.

KELLER, J.N., KINDY, M.S., HOLTSBERG, F.W., et al.
(1998). Mitochondrial manganese superoxide dismutase prevents neural apoptosis and reduces ischemic brain injury: suppression of peroxynitrite production, lipid peroxidation, and mitochondrial dysfunction. J. Neurosci. 18, 687-697.

KERMER, P., KLOCKER, N., and BAHR, M. (1999a). Longterm effect of inhibition of ced 3-like caspases on the survival of axotomized retinal ganglion cells in vivo. Exp. Neurol. 158, 202-205.

KERMER, P., KLOCKER, N., LABES, M., et al. (1998). Inhibition of CPP32-like proteases rescues axotomized retinal ganglion cells from secondary cell death in vivo. J. Neurosci. 18, 4656-4662.

KERMER, P., KLOCKER, N., LABES, M., et al. (1999b). Activation of caspase- 3 in axotomized rat retinal ganglion cells in vivo. FEBS Lett. 453, 361-364.

KIM, Y.C., KIM, S.R., MARKELONIS, G.J., et al. (1998). Ginsenosides $\mathrm{Rb} 1$ and $\mathrm{Rg} 3$ protect cultured rat cortical cells from glutamate-inducedneurodegeneration [published erratum appears in J. Neurosci. Res. 1998;54:123]. J. Neurosci. Res. 53, $426-432$.

KITTS, D.D., WIJEWICKREME, A.N., and HU, C. (2000). Antioxidant properties of a North American ginseng extract. Mol. Cell Biochem. 203, 1-10.

KOEBERLE, P.D., and BALL, A.K. (1998). Effects of GDNF on retinal ganglion cell survival following axotomy. Vision Res. 38, 1505-1515.

LAMBIASE, A., CENTOFANTI, M., MICERA, A., et al. (1997). Nerve growth factor (NGF) reduces and NGF antibody exacerbates retinal damage induced in rabbit by experimental ocular hypertension. Graefes Arch Clin. Exp. Ophthalmol. 235, 780-785.

MEY, J., and THANOS, S. (1993). Intravitreal injections of neurotrophic factors support the survival of axotomized retinal ganglion cells in adult rats in vivo. Brain Res. 602, 304-317.

NG, T.F., SO, K.F., and CHUNG, S.K. (1995). Influence of peripheral nerve grafts on the expression of GAP-43 in regenerating retinal ganglion cells in adult hamsters. J. Neurocytol. 24, 487-496.

PEINADO-RAMON, P., SALVADOR, M., VILLEGASPEREZ, M.P., et al. (1996). Effects of axotomy and intraocular administration of NT-4, NT-3, and brain-derived neurotrophic factor on the survival of adult rat retinal ganglion cells. A quantitative in vivo study. Invest. Ophthalmol. Vis. Sci. 37, 489-500.

PIERRE, S., JAMME, I., DROY-LEFAIX, M.T., et al. (1999). Ginkgo biloba extract (EGb 761) protects Na,K-ATPase activity during cerebral ischemia in mice. Neuroreport 10, 47-51.

PORCIATTI, V., PIZZORUSSO, T., CENNI, M.C., et al. (1996). The visual response of retinal ganglion cells is not altered by optic nerve transection in transgenic mice over- 


\section{CHEUNG ET AL.}

expressing Bcl-2. Proc. Natl. Acad. Sci. U.S.A. 93, 14955 14959.

RABACCHI, S.A., BONFANTI, L., LIU, X.H., et al. (1994a). Apoptotic cell death induced by optic nerve lesion in the neonatal rat. J. Neurosci. 14, 5292-5301.

RABACCHI, S.A., ENSINI, M., BONFANTI, L., et al. (1994b). Nerve growth factor reduces apoptosis of axotomized retinal ganglion cells in the neonatal rat. Neuroscience 63, 969-973.

RANCHON, I., GORRAND, J.M., CLUZEL, J., et al. (1999). Functional protection of photoreceptors from light-induced damage by dimethylthiourea and Ginkgo biloba extract. Invest. Ophthalmol. Vis. Sci. 40, 1191-1199.

SASAKI, H., INOUE, T., ISO, H., et al. (1999). Recovery of visual behaviors in adult hamsters with the peripheral nerve graft to the sectioned optic nerve. Exp. Neurol. 159, 377-390.

SAUVE, Y., SAWAI, H., and RASMINSKY, M. (2001). Topological specificity in reinnervation of the superior colliculus by regenerated retinal ganglion cell axons in adult hamsters. J. Neurosci. 21, 951-960.

SCHNEIDER, G.E. (1969). Two visual systems. Science 163, 895-902.

SO, K.F., and AGUAYO, A.J. (1985). Lengthy regrowth of cut axons from ganglion cells after peripheral nerve transplantation into the retina of adult rats. Brain Res. 328, 349-354.

SO, K.F., and YIP, H.K. (2001) The use of peripheral nerve transplants to enhance axonal regeneration in CNS neurons, in: Axonal Regeneration in the Central Nervous System (Ingoglia NA, Murray M, eds.) Marcel Dekker, Inc.: New York, pps. 505-528.

TRIPATHI, Y.B., and PANDEY, E. (1999). Role of alcoholic extract of shoot of Hypericum perforatum Linn on lipid peroxidation and various species of free radicals in rats. Indian J Exp. Biol. 37, 567-571.

VILLEGAS-PEREZ, M.P., VIDAL-SANZ, M., RASMINSKY, M., et al. (1993). Rapid and protracted phases of retinal ganglion cell loss follow axotomy in the optic nerve of adult rats. J. Neurobiol. 24, 23-36.

VOGLER, B.K., PITTLER, M.H., and ERNST, E. (1999). The efficacy of ginseng. A systematic review of randomized clinical trials. Eur. J. Clin. Pharmacol. 55, 567-575.

VORWERK, C.K., HYMAN, B.T., MILLER, J.W., et al.
(1997). The role of neuronal and endothelial nitric oxide synthase in retinal excitotoxicity. Invest. Ophthalmol. Vis. Sci. 38, 2038-2044.

WANG, X., SAKUMA, T., ASAFU-ADJAYE, E., et al. (1999). Determination of ginsenosides in plant extracts from Panax ginseng and Panax quinquefolius L. by LC/MS/MS. Anal. Chem. 71, 1579-1584.

WEI, T., NI, Y., HOU, J., et al. (2000). Hydrogen peroxide-induced oxidative damage and apoptosis in cerebellar granule cells: protection by Ginkgo biloba extract. Pharmacol. Res. 41, 427-433.

WEN, T.C., YOSHIMURA, H., MATSUDA, S., et al. (1996). Ginseng root prevents learning disability and neuronal loss in gerbils with 5-minute forebrain ischemia. Acta. Neuropathol. (Berl.) 91, 15-22.

WU, W.R., and ZHU, X.Z. (1999). Involvement of monoamine oxidase inhibition in neuroprotective and neurorestorativeeffects of Ginkgo biloba extract against MPTP-induced nigrostriatal dopaminergic toxicity in C57 mice. Life Sci. 65, 157-164.

YAN, Q., WANG, J., MATHESON, C.R., et al. (1999). Glial cell line-derived neurotrophic factor (GDNF) promotes the survival of axotomized retinal ganglion cells in adult rats: comparison to and combination with brain-derived neurotrophic factor (BDNF). J. Neurobiol. 38, 382-390.

YIP, H.K., LU, Q., and SO, K.F. (2000). The effect of CNTF and BDNF on the survival, axonal regeneration and c-jun expression of retinal ganglion cells in adult golden hamsters. Invest. Ophthalmol. Vis. Sci. 41, S189.

YIP, H.K., and SO, K.F. (2000). Axonal regeneration of retinal ganglion cells: effect of trophic factors. Prog. Retin. Eye Res. 19, 559-575.

Address reprint requests to: Kwok-Fai So, Ph.D.

Department of Anatomy Faculty of Medicine University of Hong Kong 5 Sassoon Road Li Shu Fan Building Hong Kong, China

E-mail: hrmaskf@hkucc.hku.hk 